\title{
EXISTENCE AND REGULARITY ALMOST EVERYWHERE OF SOLUTIONS TO ELLIPTIC VARIATIONAL PROBLEMS WITH CONSTRAINTS
}

\author{
BY F. J. ALMGREN, JR. 1 \\ Communicated by François Treves, July 23, 1974
}

This is a research announcement of results [A1] the full details and proofs of which have been submitted for publication elsewhere. We study the structure of $m$ dimensional subsets of $\mathbf{R}^{n}$ which are well behaved with respect to deformations of $\mathbf{R}^{n}$ and also show the existence of such sets as solutions to geometric variational problems satisfying various constraints.

Suppose, for example, one is given several positive numbers $a_{1}, a_{2}, \cdots$, $a_{N}$ and is asked to find disjointed regions $A_{1}, A_{2}, \cdots, A_{N}$ in $\mathbf{R}^{n}$ such that $A_{i}$ has volume $a_{i}$ for each $i$ and the $n-1$ dimensional area of $S=\bigcup\left\{\right.$ Boundary $\left.\left(A_{i}\right): i=1, \cdots, N\right\}$ is as small as possible. For $n=3$ this is a common formulation of a variational problem associated with compound soap bubbles. As a variant of this problem one could set $A_{0}=\mathbf{R}^{n} \sim$ $\bigcup_{i}$ Closure $\left(A_{i}\right)$ and attempt to minimize the sum of the weighted areas of the various interfaces $\left\{\operatorname{Boundary}\left(A_{i}\right) \cap \operatorname{Boundary}\left(A_{j}\right)\right\}_{i, j}$, or perhaps the weighted integrals over these interfaces of various geometric integrands. For $n=2,3$ such minimal partitioning hypersurfaces have been the subject of numerous papers in mathematics, physics, and especially biology for the past several centuries (see, for example, [TD, Chapter 4, pp. 88-125] for a discussion and references). Among other things we give the first mathematical proof of the general existence of such surfaces. The methods are representative of those required to show the existence and regularity of solutions to a variety of geometric variational problems with constraints; e.g. capillarity problems, minimal surfaces avoiding obstacles, variational problems with partially free boundaries, etc. $53 \mathrm{C} 65$.

AMS (MOS) subject classifications (1970). Primary 49F22, 49F20; Secondary

${ }^{1}$ This research was supported in part by National Science Foundation Grant NSF-GP-3314. 
The following are the main themes of [A1].

Comparison surfaces obtained by deformations. The defining properties of a surface $S$ which is either $(\gamma, \delta)$ restricted or $(F, \epsilon, \delta)$ minimal as defined below are based on comparisons of a piece $S \cap W$ of the surface with its deformed images $\phi(S \cap W)$ under lipschitzian mappings $\phi: \mathbf{R}^{n} \rightarrow$ $\mathbf{R}^{n}$; here $W=\{x: \phi(x) \neq x\}$. We develop an extensive repertoire of such lipschitzian deformations, including a partial varifold analogue of the slicing theory of [F, 4.3], in order to establish various geometric properties of such sets. In particular we do not assume the existence of a boundary operator such as is present in the theory of integral currents [F, 4]; indeed, in many of the phenomena to which our results are applicable there seems no natural notion of such an operator. Another advantage of the deformation approach is that the methods are easily adaptable to geometric variational problems in a homotopy setting [A2].

$(\gamma, \delta)$ restricted sets. Suppose $S \subset \mathbf{R}^{n}$ is locally compact and of finite $m$ dimensional measure, $B \subset \mathbf{R}^{n} \sim S$ is closed, $1 \leqslant \gamma<\infty$, and $0<\delta<\infty$. One says that $S$ is $(\gamma, \delta)$ restricted with respect to $B$ provided $H^{m}(S) \leqslant \gamma H^{m}[\phi(S \cap W)]$ whenever $\phi, W$ are as above, $W \cap B=$ $\phi(W) \cap B=\varnothing$, and $\operatorname{diam}[W \cup \phi(W)]<\delta$; here $H^{m}$ denotes hausdorff $m$ dimensional measure over $\mathbf{R}^{n}$. In case $\gamma=1$, a $(\gamma, \delta)$ restricted set locally minimizes $m$ dimensional area (and therefore is almost everywhere a real analytic minimal submanifold of $\left.\mathbf{R}^{\boldsymbol{n}}[\mathbf{A} 3,1.7]\right)$. Intuitively one might wish to regard a $(\gamma, \delta)$ restricted set as being within factor $\gamma$ of locally minimizing $m$ dimensional area. For example, for each $i=1, \cdots, N$, Boundary $\left(A_{i}\right)$ is $(\gamma, \delta)$ restricted for appropriate $\gamma, \delta$ whenever $A_{1}, \cdots$, $A_{N}$ is a solution to a reasonable partitioning problem as above. Among the main properties of a set $S$ which is $(\gamma, \delta)$ restricted are the following: (i) $S$ is $\left(H^{m}, m\right)$ rectifiable $[\mathrm{F}, 3.2 .14(4)]$, (ii) there are positive lower bounds and finite upper bounds for the density ratios of $H^{m} L S$ at all points of $\operatorname{spt}\left(H^{m} L S\right) \sim B$ for all suitably small radii, and (iii) $S$ can be approximated in a very strong sense by a diffeomorphic image of a finite polyhedral complex of dimensions $m$ and smaller. Frequently geometric variational problems give rise to varifold solutions $V$ such that $\operatorname{spt}\|V\|$ is a $(\gamma, \delta)$ restricted subset of $\mathbf{R}^{n}$, this fact depending only on upper and lower bounds for the integrand in question and not, for example, on its ellipticity [A3, 1.2]. Examples show that an additional hypothesis is required to insure a condition such as $V=|\operatorname{spt}\|V\||$; the hypothesis of ellipticity is sufficient to show this 
(compare $[\mathrm{A2}, 14(2)]$ ) as is the volume constraint in crystalline type problems [T] although the methods of proof in these two cases are totally distinct.

$(\mathbf{F}, \epsilon, \delta)$ minimal sets. Suppose $\mathbf{G}(n, m)$ denotes the grassmann manifold of all unoriented $m$ planes through the origin in $\mathbf{R}^{n}, F: \mathbf{R}^{n} \times \mathbf{G}(n, m)$ $\rightarrow \mathbf{R}^{+}$is continuous, $\epsilon: \mathbf{R}^{+} \rightarrow \mathbf{R}^{+}$is nondecreasing with $\lim _{r \downarrow 0} \epsilon(r)=0$, and $0<\delta<\infty$. One says that $S$ is $(\mathbf{F}, \epsilon, \delta)$ minimal with respect to $B$ provided $S$ is $(\gamma, \delta)$ restricted with respect to $B$ (for some $\gamma$ ) and $\mathbf{F}^{p}(S \cap W) \leqslant[1+\epsilon(r)] \mathbf{F}^{p}[\phi(S \cap W)]$ whenever $\phi, W$ are as above, $p \in$ $S \cap W, r=\operatorname{diam}[W \cup \phi(W)] \leqslant \delta$, and

$$
\mathbf{F}^{p}(T)=\int_{x \in T} F\left(p, \operatorname{Tan}^{m}\left(H^{m} L T, x\right)\right) d H^{m} x \quad \text { for } T=S \cap W, \phi(S \cap W) .
$$

Typically if $S$. is a $(\gamma, \delta)$ restricted set which is a solution to a geometric variational problem with constraints associated with $F$ and if $F$ is lipschitzian, then almost all of $S$ will be $\left(F, \epsilon, \delta^{\prime}\right)$ minimal with respect to a suitable $B$; in particular, the deformations $\phi$ need not respect the constraints. Somewhat surprisingly the choices of $\epsilon, \delta^{\prime}$, and $B$ seem to depend on the particular solution and are not determined $a$ priori. The most important fact about $(F, \epsilon, \delta)$ minimal sets and the single most important result of [A1] is the following. Suppose $F$ is elliptic and of class 3,

$$
\int_{0}^{1} t^{-(1+\alpha)} \epsilon(t)^{1 / 2} d t<\infty \text { for some } 0 \leqslant \alpha<1,
$$

and $S$ is $(\mathrm{F}, \epsilon, \delta)$ minimal with respect to $B$. Then there exists an open set $U \subset \mathbf{R}^{n}$ such that $H^{m}(S \sim U)=0$ and $S \cap U$ is a continuously differentiable $m$ dimensional submanifold of $\mathbf{R}^{n}$. In case $0<\alpha<1$ then $S \cap$ $U$ is locally hölder continuously differentiable with exponent $\alpha$. These hypotheses and conclusions, incidentally, do not imply that $S \cap U$ locally can be represented as the graph of a function which satisfies any of the various euler equations associated with $F$. For example, if $M: \mathbf{R}^{n} \times \mathbf{G}(n, m) \rightarrow\{1\}$ denotes the $m$ dimensional area integrand and if $S$ is any class 2 compact $m$ dimensional submanifold of $\mathbf{R}^{n}$, then $S$ is $(\mathbf{M}, \epsilon, \delta)$ minimal with respect to $\partial S$ whenever $\delta$ is sufficiently small and $\epsilon$ is the restriction of a linear mapping of sufficiently large norm.

\section{REFERENCES}

[A1] F. J. Almgren, Jr., Existence and regularity almost everywhere of solutions to elliptic variational problems with constraints, 294 pp. (preprint).

[A2] - The structure of limit varifolds associated with minimizing sequences of mappings, Symposia Matematica (to appear). 
[A3] - Existence and regularity almost everywhere of solutions to elliptic variational problems among surfaces of varying topological type and singularity structure, Ann. of Math. (2) 87 (1968), 321-391. MR 37 \#837.

[F] H. Federer, Geometric measure theory, Die Grundlehren der math. Wissenschaften, Band 153, Springer-Verlag, New York, 1969. MR 41 \#1976.

[T] J. Taylor, Unique structure of solutions to a class of nonelliptic variational problems, Proc. Sympos. Pure Math., vol. 27, Amer. Math. Soc., Providence, R. I. (to appear).

[TD] D'A. Thompson, On growth and form, abridged edition, Cambridge Univ. Press, New York, 1961. MR 23 \#B1601.

DEPARTMENT OF MATHEMATICS, PRINCETON UNIVERSITY, PRINCETON, NEW JERSEY 08540 